\title{
Augmented Lifecycle Space for traceability and consistency enhancement
}

\author{
József Klespitz, EKIK Physiological Control Group \\ John von Neumann Faculty of Informatics, \\ Óbuda University \\ Budapest, Hungary \\ klespitz.jozsef@phd.uni-obuda.hu
}

\author{
Miklós Bíró, Rigorous Methods in Software \\ Engineering \\ Software Competence Center Hagenberg \\ and Johannes Kepler University \\ Hagenberg/Linz, Austria \\ miklos.biro@scch.at
}

\author{
Levente Kovács, EKIK Physiological Control Group \\ John von Neumann Faculty of Informatics, \\ Óbuda University \\ Budapest, Hungary \\ kovacs.levente@nik.uni-obuda.hu
}

\begin{abstract}
In software development application lifecycle management (ALM) systems are used to support the development process. As these products are tailored for best fitting the applied programs and their actual usage is diverse. Often, this means that products of different vendors are applied, which reduce the reachability between different artefacts and the overall consistency of the system. In this paper we are analyzing the applicability of Augmented Lifecycle Space as a new approach. This approach provides the capability to enhance the traceability and consistency while reducing human effort through automation both in homogeneous and heterogeneous tool environments.
\end{abstract}

Keywords - application lifecycle management, development process improvement, open services for lifecycle collaboration, tool interoperability, tool integration

\section{INTRODUCTION}

Mature development processes are inevitable for reliable and high quality software. This is especially important in safety-critical developments and in developments with high financial impact (such in banking or in telecommunication). To obtain mature processes different management techniques are applied which are supported by application lifecycle management (ALM) systems. ALM system not only helps in supervision and interaction between consecutive software versions and releases but they are capable to track test status, provide key process indicators and support documentations as well [1]. In other words, ALM system has to provide information for management and responsible people (governance), it has to present the aptitude of development processes for assessors and business partners (development) but it has to reduce the documentation burden and work effort (operation) as discussed in [2].

Establishment of a new ALM system or improvement of an existing one is always difficult, because every company has to consider its unique needs and possibilities for best fitting. The collected preferences has to be matched with the offers of various vendors and it is practical to prove feasibility as well [3] [4]. Comparison of some vendors can be seen on Fig. 1. depending on their completeness of vision and their ability to execute.

Companies can choose from comprehensive, general systems, and programs solving particular problems. Depending on the choice(s) this result one or more components, however, systemic management requires customization. In this paper we wish to present the applicability of method Augmented Lifecycle Space. This approach is based on Open Services for Lifecycle Collaboration (OSLC) and it can be used to increase transparency throughout the system. Moreover, in this Augmented lifecycle space the missing or incorrect relationships can be highlighted and the elimination process of these problems can be generated automatically. This versatile technique helps not only the management and developers in the development process, but it highly supports the assessment process as well.

In this paper we present how this technique can be used in a theoretical system and we demonstrate the enhancement it provides. The main targets of this approach are companies with heterogeneous tool environment. Although, more integrated system can be improved this way, but the benefits are less explicit in such cases. 


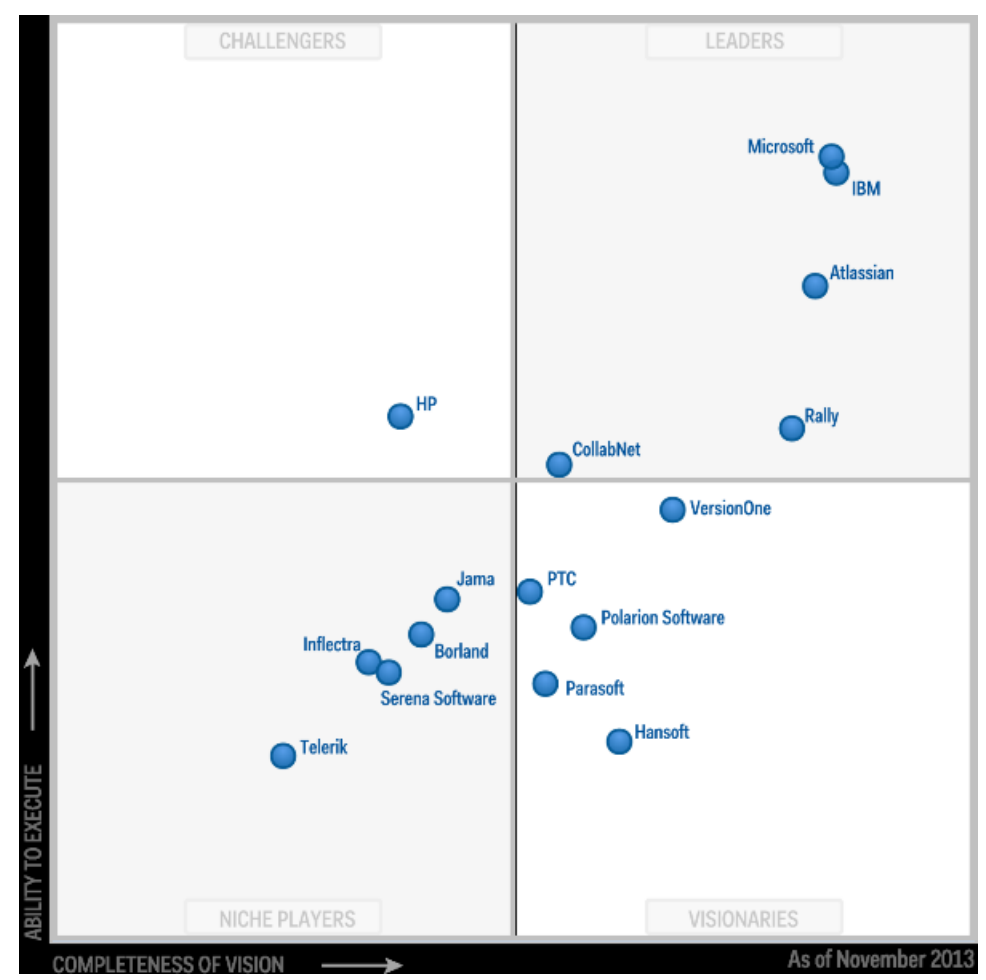

Fig. 1. ALM providers and their capability [31] with completeness of vision on horizontal axis and their ability to execute on the vertical axis

\section{IMPORTANCE OF IMPROVEMENT}

Nothing shows importance of traceability better than standard IEC 61508 recommends [5] the use of forward and backward traceability (together bilateral or bidirectional traceability) for Safety Integrity Level (SIL) 1 and 2 , and it is highly recommended for SIL 3 and 4 applications. On the other hand, it is known [3] that 50$60 \%$ of software defects originates from requirement development, where the rate of leakage is $53 \%$ in the requirements phase and $68 \%$ in the design phase [6].

Despite these facts, a significant proportion of people in charge of software development see traceability as a mandatory burden or as a useful but cumbersome duty [79]. In line with this various fields have related prescriptions regarding traceability. In generic software development the Capability Maturity Model Integration (CMMI) [10] and standard ISO/IEC 15504 requires [11] traceability in development. In safety-critical software developments DO-178C has similar prescription for airborne systems [12], while ISO 26262 for road vehicles [13]. Moreover, Automotive SPICE [14] and MDevSPICE [15] has to be mentioned as further directives with traceability related ordinance for cars and medical devices (where SPICE stands for Software Process Improvement and Capability dEtermination).

Current paper focuses mostly on development of medical devices. In this domain the Medical Device Directive (MDD) of European Council [16] and guidance of the Food and Drug Administration (FDA) of the United States [17] are the most noticeable guidelines which involves traceability. Furthermore, standards IEC 62304 [18], ISO 14971 [19], IEC/TR 80002 [20], and ISO 13485
[21] has to be mentioned. More can be read about traceability in [22].

Consistency of system was emphasized first by the Automotive SPICE published in 2015 [14]. They have defined consistency that the examined contents and semantics should not contradict each other. Although, we focus on medical development the benefits and the possible appearance in medical standards and directives make worthy to use it already in this domain. Fig. 2. helps understanding how traceability and consistency can be fulfilled throughout the development: in this classical Vmodel it is clearly marked that in the decomposition phase (left side) requirements at adjacent levels has to be connected (blue arrows). This way the origin of requirements can be tracked and their relevance is known. Naturally, the requirements has to have their counterpart in testing (to be more precise - during verification and validation). These connection altogether makes sure that the customer needs are fulfilled (and only those). Moreover, not only connection are necessary for the artifacts (traceability), but their content has to be coherent without contradiction (consistency, red arrows).

It is important to point out that assessors face relevant challenges when analyzing traceability and consistency. Firstly, assessment can be done only on a closed ALM system. The presentation of traceability is cumbersome (e.g. traceability matrix), which makes complete inspection impossible. Furthermore, the assessors has to navigate among many widgets (with some custom application as well). Augmented Lifecycle Space helps to bridge the aforementioned problems both on development and assessment side. 


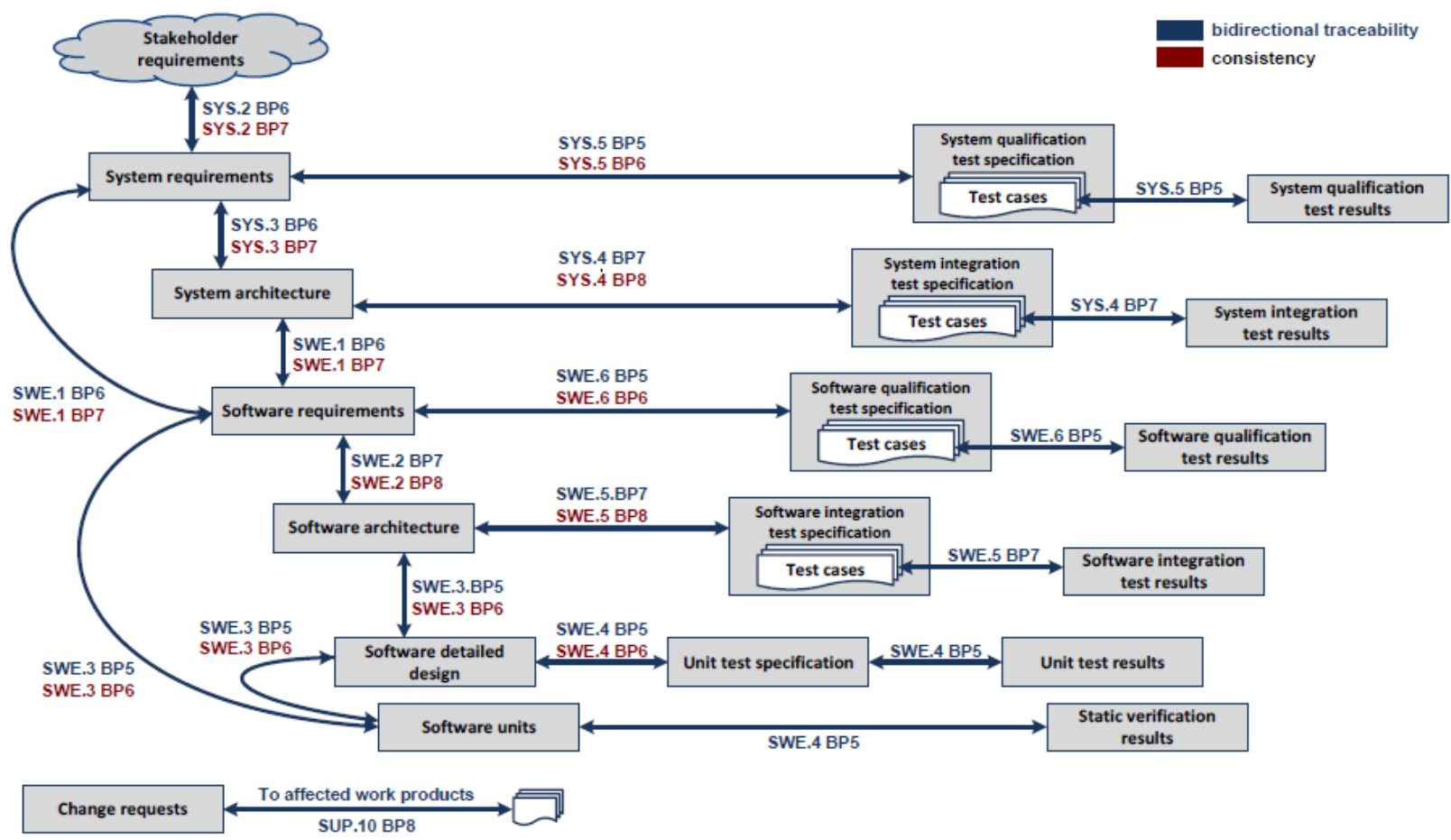

Fig. 2. Traceability (blue) and consistency (red) when using V-model [14]

\section{NEED OF CROSS-TOOL INTEROPERABILITY}

As we have already mentioned, ALM systems standing in our focus consist various, often incompatible components. Using different tools can be caused by many factors: A company may choose not to depend on a certain vendor and sometime the unique needs can only be matched with the offers of different vendors. Similarly, heterogeneity might be resulted if the tool system was established incrementally over time. Moreover, there is qualm about the loss of accumulated intellectual property. ALM systems store tremendous amount of precious information which might be damaged or lost in case of migration. Therefore, companies, most of the time, insist keeping well-tried solutions to avoid such scenarios. Finally, people have to overcome their habits and get used to new programs which can be cumbersome even for developers. The above mentioned problems coincide with our experience.

In a diversified environment, traceability, consistency and usability issues are naturally amplified. In cross-tool relationships, where direct connections are nonexistent, (e.g. requirements and their tests may be present in different tools) aging will erode traceability, and can lead to inconsistencies. Thus, it is of upmost importance to create direct connections between artifacts in order to maintain traceability and enable consistency analysis. Furthermore, replication can be eliminated which further improves transparency and decreases the risk of introduced mistakes.

To eliminate these weaknesses cross-tool interoperability has to be established. By having a system, where all of the components are capable to communicate with each other, the above mentioned problems cannot occur. Direct links between different artifacts help to have clear traceability, where inspections of consistency and traceability can be easily run automatically. Furthermore, using the known relationships between the artifacts workflow can be created automatically for changes or fault corrections (where fault correction means both bug fixing and restoring consistency). What is more, in case of redundant entries or straightforward relationship certain artifacts can be generated. For example if a revision control system is connected to the tool chain the comment field can be used to describe the modification, explain and store the content in the issue tracking system and even the necessary automatic tests can be triggered. As a possible solution we suggest to use Augmented Lifecycle Space (ALS) which provides unique approaches to overcome these problems. The idea of ALS is discussed later in this paper.

\section{LINKED DATA FOR INTEROPERABILITY}

The idea to use interoperability in not new [22] [23]. However, we wish to point out the faults of previous concepts and establish a more effective model. The ALS is based on the recognition missing links and artifacts and their related processes. The original Lifecycle Space can be found a priori in the ALM system. The goal is to expand this Lifecycle Space to eliminate the defects of the original one.

To get the ALS the following steps are suggested: The existing artifacts in the tool environment have to be categorized and their relationships have to be explored. The missing artifacts have to be created (automatically) according to the traceability requirements (and consistency requirements) of the used model. The augmentation of space means this process- to complete the system with missing objects and links. Finally, a workflow can be generated automatically, by analyzing 
the missing artifacts and their environment, to overcome these defects.

The benefits of this approach are twofold. It is trivial that this method can be used to complete and correct the existing ALM system. On the other hand, the ALS can be used for development planning. If a non-existent object is created only in the ALS its effect can be analyzed. The relationships and the related workflow can be generated automatically for this "virtual" object and the workload can be clearly calculated. This is ultimately useful for planning introduction of a new feature or analyzing the effect of a high level requirement change.

The Open Services for Lifecycle Collaboration (OSLC) provides an excellent platform to realize ALS [25], as Fig. 3 shows. It uses web architecture, and creates linking between artifacts via linked data. Single entries

\section{CONCLUSION AND FURTHER WORK}

The need of ALM systems in modern software development is unquestionable. They support both the management in governance, the development by organizing information, and operation by providing the necessary information. The traceability and consistency is upmost important for safety-critical developments, both for developers and assessors.

Nowadays many vendor is competing for the companies. Heterogeneous environment can be caused by unique needs, historical reasons, or to stay independent of vendors. The various programs raise a challenge for everyone. It is not only hard to keep updated databases which are not directly connected, but the developers has to use many different environment as well. Furthermore, the assessors face unique problems with each assessed development. In order to enhance traceability, consistency, and usability it is practical to integrate the used platforms.

Nowadays, the most advanced technology is to use web architecture to create connection between the artefacts. The use of linked data prevents many problems of the previous solutions such as redundant data storage or aging of databases. Open Services for Lifecycle can be kept as they can be reached via URIs without copying. The used standard is accepted by many important vendors. OSLC has become more popular in the last years and a growing number of adaptors can be found on the market developed by the vendors themselves (e.g. IBM developed adaptors for Atlassian JIRA or Git) or by third party (e.g. Tasktop).

Applicability of OSLC is researched and proofed to be effective [26-28]. However, the existing solutions do not exploit the full potential. Therefore, we wish to expand interoperability for further benefits. We wish to prove the capability of the aforementioned ideas through a case study. The analyzed ALM system will consist four independent tool for requirement management, test management, workflow management, and one for providing OSLC adapter (relationship management).

collaboration (OSLC) provides a widely accepted standard for this.

In this paper we have presented the idea of Augmented Lifecycle Space. By using this new approach we are expecting to:

- have better traceability and consistency,

- explore traceability and consistency defects automatically,

- generate workflow automatically to repair these defects,

- generate workflow automatically if a requirements is changed or created,

- integrate revision control system to eliminate redundant documentation,

- show every necessary information for the developers in the development environment,

- be able to analyze the effect of a new feature or high level requirement change.

A case study will be carry out on an ALM system with tools from four different vendors. The integration will be created with the help of Tasktop Sync, which will provide the framework for this research and provides the possibility to access every necessary information out of databases.

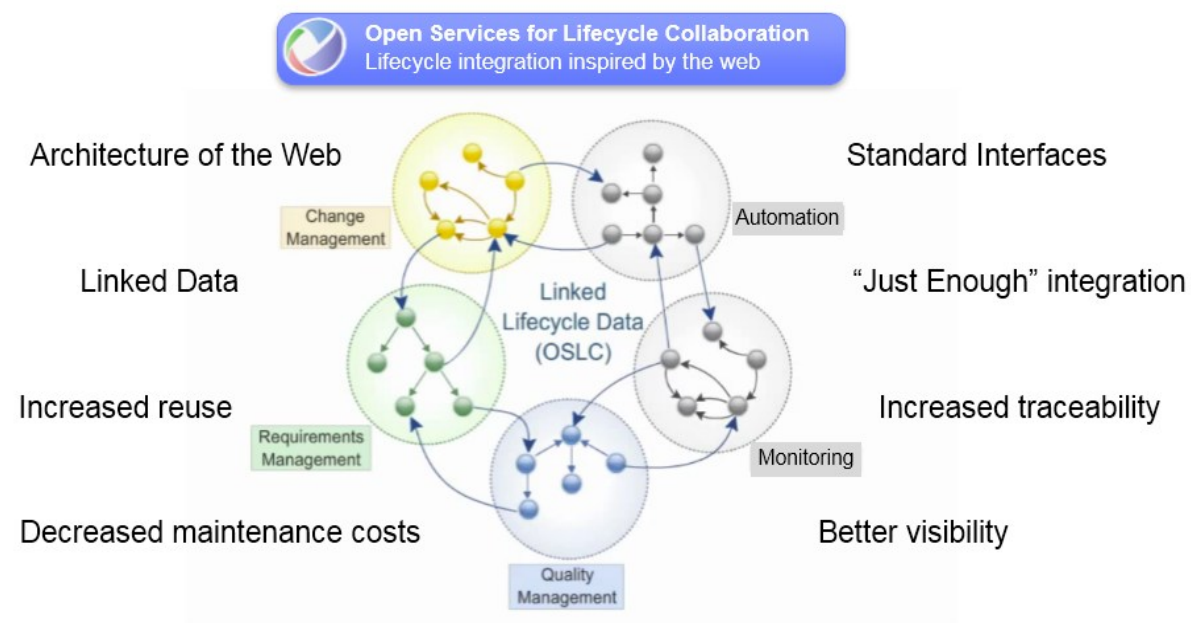

Fig. 3. Benefits of OSLC 


\section{ACKNOWLEDGMENT}

The authors are grateful for the support of Research and Innovation Center of Óbuda University. The work is supported by the European Research Council Starting Grant ERC-StG 679681.

The research reported in this paper has been supported by the Austrian Ministry for Transport, Innovation and Technology, the Federal Ministry of Science, Research and Economy, and the Province of Upper Austria in the frame of the COMET center SCCH.

\section{REFERENCES}

[1] S. Chanda, F. Damien. Application Lifecycle Management. In Beginning ASP. NET 4.5 Databases. Apress, 2013. p. 235-249.

[2] D. Chappell. December 2008. What is application lifecycle management? [Online] Available: http://www.davidchappell.com/WhatIsALM--Chappell.pdf

[3] J. Klespitz, M. Biró, L. Kovács. Aspects of improvement of software development lifecycle management. In 16th IEEE International Symposium on Computational Intelligence and Informatics (CINTI), 2015: p. 323-327.

[4] J. Brown. January 2016. A PLM systems comparison of the industry's top tools In Techtarget [Online]. Available: $\mathrm{http} / /$ searchmanufacturingerp.techtarget.com/feature/A-PLMsystems-comparison-of-the-industrys-top-tools

[5] IEC, IEC-61508: Functional Safety of Electrical/ Electronic/Programmable Electronic Safety-related Systems. 2010, Geneva, Switzerland.

[6] F. McCaffery, V. Casey, M. S. Sivakumar, G. Coleman, P. Donnelly, J. Burton: Medical device software traceability. In Software and Systems Traceability, Springer London, 2012 pp. 321-339.

[7] J. Capers: Software Quality in 2011: A Survey of the State of the ART. In Capers Jones \& Associates LLC., 2011

[8] P. Nistala, P. Kumari: Establishing content traceability for software applications: An approach based on structuring and tracking of configuration elements. In 7 th Workshop on Traceability in Emerging Forms of Software Engineering (TEFSE), 2013

[9] E. Bouillon, P. Mäder, I. Philippow: A survey on usage scenarios for requirements traceability in practice. In 19th International Working Conference on Requirements Engineering: Foundation for Software Quality (REFSQ), Vol. 7830, LNCS, Springer, 2013, pp. 158-173.

[10] SEI, CMMI® for Development, Version 1.3. 2010, Software Engineering Institute

[11] ISO/IEC, ISO/IEC 15504: Information Technology - Process Assessment. 2011: Geneva, Switzerland.

[12] RTCA/DO, RTCA/DO-178C: Software Considerations in Airborne Systems and Equipment Certification, 2012.

[13] ISO, ISO 26262: Road vehicles - Functional safety. 2011, Geneva, Switzerland.
[14] Automotive, S. I. G., VDA QMC Working Group 13 Automotive SPICE Process Assessment / Reference Model, v3.0, 2015:470.

[15] Celtic MedTech Ltd., MDevSPICE, 2015, Dundalk, Ireland.

[16] .European Commission, Directive 93/42/EEC of the European Parliament and of the Council concerning medical devices, in $\mathrm{OJ}$ o L 247 of 2007-09-21. 1993: European Commission, Brussels, Belgium.

[17] FDA. Chapter I - Food and drug administration, department of health and human services subchapter $H$ - Medical devices, Part 820 - Quality system regulation [Online]. Available: http://www.accessdata.fda.gov/scripts/cdrh/cfdocs/cfcfr/CFRSearc h.cfm?CFRPart=820.

[18] IEC, IEC 62304: Medical Device Software - Software Life-Cycle Processes. 2006, IEC: Geneva, Switzerland

[19] ISO, ISO 14971 - Medical Devices - Application of Risk Management to Medical Devices 2009, ISO: Geneva, Switzerland.

[20] IEC, IEC TR 80002-1 - Medical Device Software - Part 1: Guidance on the Application of ISO 14971 to Medical Device Software. 2009, IEC: Geneva, Switzerland.

[21] ISO, ISO 13485: Medical Devices - Quality Management Systems - Requirements for Regulatory Purposes. 2003, ISO: Geneva, Switzerland.

[22] J. Cleland-Huang, O. Gotel, A. Zisman. Software and systems traceability. Vol. 2(3). Springer, 2012.

[23] W Zhang, V. Leilde, B. Moller-Pedersen, J. Champeau, C. Guvchard. Towards tool integration through artifacts and roles In 19th Asia-Pacific Software Engineering Conference (APSEC), 2012, vol. 1, IEEE, 2012, p. 603-613.

[24] A. Fisher, B. Williams, S. Matthews. Linked Lifecycle DataConnecting Distributed Engineering Data and harvesting its inherent value. In INCOSE International Symposium, vol. 23(1), 2013, p. 331-345.

[25] OSLC Core Specification Workgroup. OSLC core specification version 2.0. Open Services for Lifecycle Collaboration, 2013.

[26] B. K. Aichernig, K. Hormaier, F. Lorber, D. Nickovic, R. Schlick, D. Simoneau. S. Tiran. Integration of reauirements engineering and test-case generation via OSLC. In 14th International Conference on Quality Software (QSIC), IEEE, 2014, p. 117-126.

[27] M. Saadatmand, A. Bucaioni. OSLC Tool Integration and Systems Engineering--The Relationship between the Two Worlds. In 40th EUROMICRO Conference on Software Engineering and Advanced Applications (SEAA), IEEE, 2014, p. 93-101.

[28] M. Saadatmand, M. Sjödin. Tool integration using OSLC. master thesis, 2013.

[29] Tasktop Technologies, 2016. Task Linking Configuration - User Guide [Online]. Available:

http://help.tasktop.com/help/index.jsp?topic=\%2Fcom.tasktop.syn c.help \%2Fuserguide\%2FTask-Linking-

Configuration.html\&cp $=1 \_0 \_7$

[30] G. Regan, M. Biro, F. Mc Caffery, K. McDaid, D. Flood. A traceabilitv process assessment model for the medical device domain. In Systems, Software and Services Process Improvement, Springer Berlin Heidelberg, 2014, p. 206-216.

[31] S Hastie, 23 February 2015. Gartner and Software Advice examine Agile Lifecycle Management Tools In InfoQ [Online] Available: http://www.infoq.com/news/2015/02/agile-management-tools 